\title{
La Práctica Profesional en Enfermería: retos de los estudiantes ante la nueva modalidad en línea.
}

\author{
Professional Practice in Nursing: challenges for students in the new online \\ modality.
}

\author{
Susana B. Reyes Torres ${ }^{a}$, Rocío B. Mayorga Ponce ${ }^{b}$, María L. Sánchez-Padilla ${ }^{c}$, Reyna C. \\ Jiménez-Sánchez ${ }^{d}$, Olga R. Chávez-Flores ${ }^{e}$ y Rosa M. Baltazar-Téllez ${ }^{f}$
}

\begin{abstract}
:
For nursing students, like other bachelor's degrees in the area of health, it is of the utmost importance to put into practice the knowledge acquired in the classroom; hence, Professional Practices play a fundamental role for students to employ and strengthen the skills gained throughout their academic training. However, the situation that at the moment concern to society worldwide has forced the school authorities to carry out Professional Practices of nursing students in a virtual modality as a way to safeguard the health of the university community and society in general..
\end{abstract}

Keywords:

Professional Practice, Nursing, Challenge, Education, Virtual

\section{Resumen:}

Para los estudiantes de enfermería, al igual que las demás licenciaturas del área de la salud, resulta de suma importancia llevar a la práctica el conocimiento adquirido en las aulas; de ahí que las Prácticas Profesionales jueguen un papel fundamental para que los estudiantes empleen y fortalezcan las competencias ganadas a lo largo de su formación académica. No obstante, la situación que en estos momentos ataña a la sociedad a nivel mundial ha obligado a las autoridades escolares a llevar a cabo las Prácticas Profesionales de los estudiantes en enfermería en una modalidad virtual como una manera de salvaguardar la salud de la comunidad universitaria y la sociedad en general.

Palabras Clave:

Práctica Profesional, Enfermería, Reto, Educación, Virtual

\section{Introducción}

La práctica de enfermería se conceptualiza como, el conjunto de aspectos cognoscitivos, de comportamiento y experiencias, que lleva a cabo una enfermera/o practicante $o$ en ejercicio al proporcionar cuidados de salud al usuario. El plan de estudios de la Licenciatura en Enfermería se estructura desde un enfoque interdisciplinar en conjunto con un modelo curricular integral; teniendo como elementos integradores a las competencias que pueden ser definidas como la integración de contenidos para lograr el desempeño profesional satisfactorio,

\footnotetext{
Autor de Correspondencia, Universidad Autónoma del Estado de Hidalgo, https://orcid.org/0000-0002-9830-2589, Email: re375087@uaeh.edu.mx

b Universidad Autónoma del Estado de Hidalgo, https://orcid.org/0000-0003-3544-7171, Email: rmponce@uaeh.edu.mx

c Universidad Autónoma del Estado de Hidalgo, https://orcid.org/0000-0001-7621-0993,Email: maria_sanchez2789@uaeh.edu.mx

d Universidad Autónoma del Estado de Hidalgo, https://orcid.org/ 0000-0001-9264-8514, Email: jimenezs@uaeh.edu.mx

e Universidad Autónoma del Estado de Hidalgo, https://orcid.org/ 0000-0001-9479-9834, Email: oflores@uaeh.edu.mx

f Universidad Autónoma del Estado de Hidalgo, https://orcid.org/0000-0002-2168-7564, Email: rbaltazartellez@yahoo.com.mx
} 
establece el hilo conductor entre el conocimiento cotidiano, académico y científico. ${ }^{(1)}$

Además de consolidar la formación integral de los estudiantes su retroalimentación y actualización, las Prácticas Profesionales (PP) buscan la vinculación del estudiante de enfermería con los escenarios laborales permitiendo aplicar los conocimientos adquiridos para la toma de decisiones sobre el cuidado de acuerdo a los componentes fundamentales de la enseñanza ${ }^{(2)}$; como lo establece el diseño curricular de la Educación Superior permitiendo a los estudiantes la apropiación de modos de actuación profesional en lo académico, práctico o laboral e investigativo preparando su inserción en el ámbito laboral. ${ }^{(1)}$

Tras la crisis sanitaria causada por la COVID-19 nuestra máxima casa de estudios con el compromiso de brindar una educación de calidad y ante los cambios que demandaban las normas sanitarias llevo a cabo la implementación del modelo Blended Learning (BLearning) desde el segundo periodo escolar de 2020; donde a través de un sistema híbrido se buscó emplear las fortalezas tanto de la modalidad presencial como de la modalidad en línea priorizando el uso de las Tecnologías de la Información y Comunicación (TIC) para brindar a la comunidad universitaria una enseñanza autónoma, colaborativa y personalizada. ${ }^{(3)}$

\section{Desarrollo}

Si bien es cierto que la implementación de los recursos tecnológicos han permitido la mejora en el aprendizaje y el desarrollo de destrezas de los estudiantes de enfermería (un claro ejemplo es el Centro Latinoamericano de Educación Médica Por Simulación: CLEMPS, que permite la simulación del entorno hospitalario mediante modelos robotizados y electrónicos con la finalidad de disminuir los errores más comunes en la práctica clínica) ${ }^{(4)}$, la aplicación de estos recursos se mantienen en evolución constante asi como nuestra familiarización en su uso; por lo tanto, la repentina aplicación del modelo de aprendizaje virtual ha representado un gran reto tanto para los docentes como para los alumnos, por un lado los profesores buscan que esta situación no afecte la adquisición de conocimientos, capacidades y competencias de sus alumnos y por otro lado los estudiantes han tenido que lidiar con las barreras de acceso a las TIC ${ }^{(5)}$. A raíz de la pandemia resulto evidente que a pesar de los grandes avances tecnológicos que nos rodean los modelos de enseñanza no están adaptados para ser aplicados en una situación a distancia como la que se está atravesando, teniendo en cuenta que el aislamiento es necesario para mantener al mínimo el número de contagios en las aulas, por lo que se tiene que analizar las ventajas y desventajas de esta nueva modalidad para replantear los modelos de enseñanza en enfermería y mejorar la calidad de aprendizaje de los alumnos. $^{(6)}$

Investigaciones recientes han descrito la perspectiva de los estudiantes de enfermería ante la implementación de la modalidad virtual, los retos a los que se han venido enfrentado y como impactan en su aprendizaje y en la práctica profesional.

Román et al. ${ }^{(7)}$ tras la aplicación de una entrevista pudo identificar dos vertientes (como se muestra en la Figura 1) en cuanto a las experiencias de los participantes, la primera se basa en el cambio del aprendizaje presencial al virtual donde los estudiantes se enfrentaron mientras que la segunda muestra las dificultades que se presentaron en el aprendizaje.

Figura 1. Esquema de categorías y subcategorías.

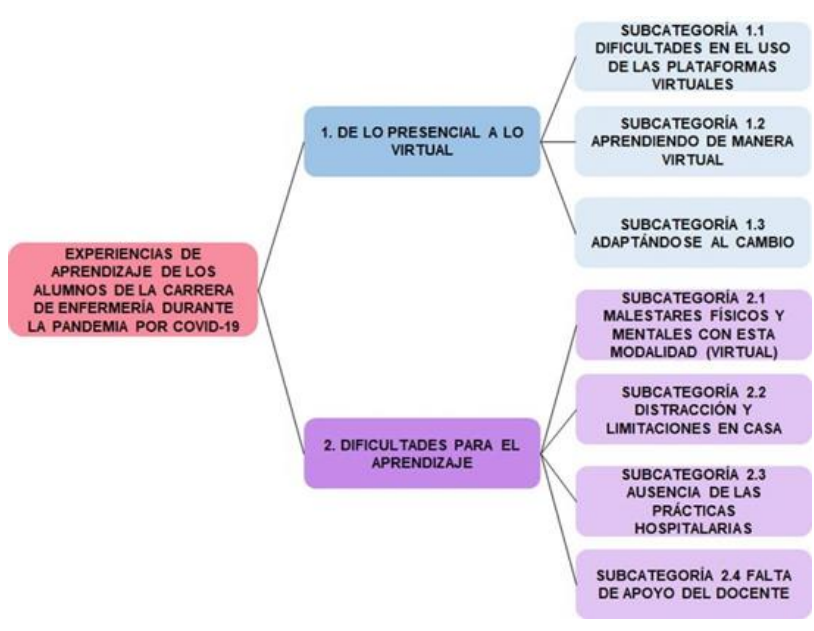

Fuente: Román et al. Experiencias de aprendizaje de los alumnos de la carrera de Enfermería durante la pandemia por COVID-19. 2021.

La dificultad en el uso de plataformas digitales fue una de las situaciones que problematizaron el aprendizaje en los alumnos no solo por el hecho del desconocimiento al utilizar dichas plataformas sino que también influían ciertas situaciones que no dependían de los participantes como la calidad del internet con el que contaban alumnos y profesores al momento de las clases virtuales, o la saturación de las plataformas por el uso en masa evitando que puedan enviar los trabajos en tiempo y aunque para algunos el uso de las plataformas no era el problema, sino más bien el acceso limitado a servicios de buena calidad. En cuanto al aprendizaje de manera virtual menciona que los alumnos han expandido su conocimiento sobre 
plataformas virtuales que desconocían y que ahora facilitan la elaboración de sus trabajos aun así no se puede dejar de lado la aparición del estrés tras pasar una larga jornada frente a los monitores sumado a la presencia de distractores que influyen en el nivel de atención de los alumnos. Para la adaptación al cambio aunque al principio no fue fácil solo basto determinado tiempo para seguir el ritmo de nuevas rutinas y poder atender todas sus clases y poder aprobar su ciclo escolar.

Es necesario recalcar que no para todos los estudiantes ha resultado fácil adaptarse a una situación como la que se vive hoy en día; los alumnos también han podido identificar un gran cambio en la relación con sus docentes y una gran barrera en el manejo de las TIC lo que obstaculiza su desempeño en las aulas virtuales; y si a esto le sumamos la aparición de distractores, pues la gran mayoría no cuenta con un espacio adecuado para tomar clases y por lo general comparten el mismo espacio y equipos con más miembros de la familia, podemos observar que existe un impacto significativo en el aprendizaje y tareas que no suponían un problema en las actividades presenciales. En vista de dichos acontecimientos se ha podido confirmar que los alumnos han tenido repercusiones a nivel físico y mental consecuencia de las largas jornadas frente a los monitores.

Si de la práctica clínica hablamos no podemos negar es una parte importante dentro de la formación de los estudiantes en enfermería, situación que es reafirmada por los participantes de dicha investigación quienes mencionan que las PP son algo imprescindible en su aprendizaje pues a pesar de contar con el conocimiento teórico el no llevar a cabo las PP genera en ellos inseguridad como futuros egresados ya que perciben una carencia de herramientas para desempeñarse en el ámbito hospitalario.

De manera similar a la investigación anterior Meléndez ${ }^{(8)}$ nos permite conocer las experiencias de alumnos de la licenciatura en enfermería referentes a las PP donde los resultados que obtuvo se dividen en las siguientes categorías Práctica como apoyo docente, Práctica en la enseñanza, Campo clínico en la formación de enfermería y Mundo de vida de los estudiantes.

Los participantes en esta investigación manifiestan que si bien la práctica puede ser usada como un recurso didáctico de los docentes para complementar la teoría también es una gran herramienta para mejorar el aprendizaje y las técnicas abordadas en clase, recalcando la gran diferencia de llevar a cabo la practica en casa en comparación a los laboratorios o simuladores. Otra situación que añoran los estudiantes más allá del reforzar su aprendizaje es la experiencia y la esencia de las prácticas en un hospital, la relación con los pacientes y el trabajo codo a codo con el equipo multidisciplinario en las instituciones de salud atribuyéndole un gran valor que antes no asignaban a las prácticas, y aunque están conscientes que el aislamiento fue una medida de protección necesaria para ellos y sus familias no dejan de sentir preocupación por la falta de practica lo que los ha llevado a preocuparse por su futuro, pues no saben cuándo volverán a pisar de nuevo el hospital y si se encuentran lo suficientemente preparados para llevar a cabo su labor, temen por el rechazo del personal de enfermería a cargo de los servicios hospitalarios al querer participar en procedimientos o peor aún, no poder realizar ciertos procedimientos debido a la carencia en sus habilidades técnicas; por lo que comenzaron a reflexionar sobre su futuro laboral y el impacto que tienen las PP sobre este, tienen un gran sentido de responsabilidad ya que como futuros egresados serán los encargados cuidar y prevenir los problemas de salud en la sociedad.

\section{Conclusión}

Todo lo planteado hasta ahora nos lleva a concluir que la aparición de la COVID-19 trajo grandes cambios para el sistema educativo, en especial para las carreras de las ciencias de la salud; que mantienen un enfoque interdisciplinar a través del método teórico-práctico dentro de su programa de estudios, repercutiendo de manera negativa en el aprendizaje de los estudiantes de enfermería quienes expresan que el mayor reto derivado de este aislamiento va más allá de las fallas de conexión, los distractores de su entorno y las afectaciones físicas tras largas jornadas virtuales: la ausencia de las Prácticas Profesionales. Situación que los llevo a dimensionar la importancia que estas tienen en el desarrollo de sus habilidades como futuros profesionales y que tras más de un año y medio sin poner en práctica sus conocimientos teóricos genera en ellos incertidumbre sobre su futuro en las instituciones médicas y si serán capaces de brindar el cuidado que requieren los pacientes. Por lo tanto se espera que las Universidades impulsen proyectos que permitan a los alumnos compensar el tiempo fuera de los laboratorios y sean capaces de desarrollar las competencias necesarias para enfrentar cualquier situación en el ámbito hospitalario; además de seguir adaptando el uso de la tecnología a su favor y en pos de un plan educativo que no se vea limitado por la aparición de emergencias sanitarias, pues sin duda alguna las Prácticas Profesionales son una herramienta indispensable para el aprendizaje de los estudiantes de enfermería permitiéndoles no solo crecer como 
profesionales sino como seres humanos a través de la verdadera esencia de la enfermería, el cuidado.

\section{Referencias}

[1] Mayorga R, Trejo C, Martínez A. Proyección de la Práctica Virtual Profesional de la Licenciatura en Enfermería $8^{\circ}$ Semestre. México: Área Académica de Enfermería UAEH; 2020.

[2] Vargas M. Departamento de Enfermería: Prácticas Profesionales. [Internet]. México: Universidad de Sonora; 2017. [Citado el 10 de octubre de 2021]. Disponible en: https://enfermeria.unison.mx/practica-profesional/

[3] Zamora A. B-LEARNING, Aprendizaje en tiempos de distanciamiento social. [Internet]. México: Gaceta UAEH; 2020. [Citado el 10 de octubre de 2021]. Disponible en https://www.uaeh.edu.mx/gaceta/2/numero18/agosto/b-learning.html

[4] ICSa. CLEMPS Antecedentes. [Internet]. México: UAEH. [Citado el 10 de octubre de 2021]. Disponible en: https://www.uaeh.edu.mx/campus/icsa/clemps/acerca_de_nosotros.ht $\mathrm{ml}$

[5] López F. Retos de la enseñanza virtual en las facultades de Enfermería. [Internet]. España: ELSEVIER; 2020. [Citado el 10 de octubre de 2021]. Disponible en: https://www.elsevier.com/eses/connect/enfermeria/retos-de-la-ensenanza-virtual-en-las-facultadesde-enfermeria

[6] Costa R, Lino M, Souza A, Lorenzini E, Fernandes G, Brehmer L, et al. Enseñanza de enfermería en tiempos de COVID-19: ¿cómo reinventarla en este contexto?. Texto Contexto Enferm [Internet]. 2020 [Citado el 10 de octubre de 2021]; 29 (e20200202): 1-3. Disponible en: https://doi.org/10.1590/1980-265X-TCE-2020-0002-0002

[7] Román N, Morales A, Juárez M, Godínez M, Regalado L, Cuevas L. Experiencias de aprendizaje de los alumnos de la carrera de Enfermería durante la pandemia por COVID-19. Ocronos [Internet]. 2020 [Citado el 10 de octubre de 2021]; 4(7): 107. Disponible en: https://revistamedica.com/aprendizaje-alumnos-enfermeriapandemia/\#Metodologia

[8] Meléndez S. La importancia de la práctica en la formación de enfermería en tiempos de Covid-19: experiencias de alumnos. Dilemas contemp. educ. política valores [Internet]. 2020 [Citado el 10 de octubre de 2021]; 8 (spe5): $1-14 . \quad$ Disponible en: https://doi.org/10.46377/dilemas.v8i.2479 